\title{
Fístula aortoentérica como causa de hemorragia digestiva en tres pacientes adultos
}

\author{
A. ACOSTA, R. KARATANASOPULOZ PINTOS, C. HAYDAR, G. LEVY, C. MARTÍN Y M. PAIZ \\ Servicio de Terapia Intensiva. Hospital José Ramón Vidal. Corrientes. Argentina.
}

El propósito de esta presentación es comunicar el caso de tres pacientes adultos con fístula aortoentérica, cuya forma de presentación inicial fue dolor abdominal y sangrado digestivo. Destacamos la muy baja incidencia de esta patología como causa de hemorragia digestiva, su elevada mortalidad y la necesidad de una alta sospecha clínica para el diagnóstico. Discutimos las diferentes localizaciones de las fístulas aortoentéricas, los probables mecanismos fisiopatológicos que las generan y su elevada asociación con la presencia de prótesis vasculares.

PALABRAS CLAVE: fístula aortoentérica, hemorragia digestiva.

\section{AORTOENTERIC FISTULA AS CAUSE OF DIGESTIVE BLEEDING IN THREE ADULT PATIENTS}

The purpose of this presentation is to report three adult patients with aortoenteric fistula whose initial presentation was abdominal pain and digestive bleeding. We stress the low incidence of this disease as a cause of digestive bleeding, its high mortality and the need of high clinical suspicion for its diagnosis. We discuss the different sites of the aortoenteric fistulas, probable physiopathological mechanisms that generate them and their elevated association with the presence of vascular prostheses.

KEY WORDS: aortoenteric fistula, digestive bleeding.

Correspondencia: Dr. R. Karatanasopuloz Pintos.

Terapia Intensiva.

Hospital José Ramón Vidal.

C/ Necochea, 1050.

3400 Corrientes, Argentina.

Correo electrónico: rkarata@yahoo.com.ar

Manuscrito aceptado el 12-I-2006.

\section{INTRODUCCIÓN}

La fístula aortoentérica (FAE), definida como la comunicación entre la aorta y un asa intestinal, es una causa rara de hemorragia digestiva, que conlleva una elevada mortalidad, por lo que requiere una alta sospecha clínica para el diagnóstico y una conducta quirúrgica urgente. Desde su primera descripción se han reportado alrededor de 250 casos de FAE primarias ${ }^{1}$, y la tasa de mortalidad oscila entre el $30 \%-85 \%{ }^{2}$.

Se llaman fístulas aortoentéricas primarias a aquellas que son consecuencia de patologías aórticas como aneurismas (90\% de los casos) o procesos patológicos de vecindad que incluyan al tubo digestivo. El segmento más frecuentemente involucrado en el aparato digestivo es el duodeno en un $80 \%$ de los casos, especialmente la tercera porción $(51 \%)$, debido a la posición fija del duodeno en el retroperitoneo, aunque puede localizarse en cualquier otra porción del tubo digestivo.

Las fístulas aortoentéricas secundarias son aquellas en las que encontramos factores predisponentes, tales como la colocación de prótesis vasculares, en su gran mayoría, y raramente cuerpos extraños como agujas, espinas de pescados, trauma, etc.

Presentamos tres casos de fístulas aortoentéricas ingresados en el Servicio de Terapia Intensiva del Hospital Dr. José Ramón Vidal de la ciudad de Corrientes, Argentina, cuyo diagnóstico definitivo fue realizado en la sala de cirugía durante laparotomías de urgencia.

\section{DESCRIPCIÓN DE LOS CASOS}

Caso clínico 1

Se trata de un paciente de 58 años de edad, de sexo masculino, con hipertensión arterial de más de 10 años de evolución, tratado pero no controlado. 
Como antecedente patológico relevante el paciente habia advertido una masa abdominal palpable y pulsátil en el último mes. Diez días antes del ingreso en nuestro centro presentó dolor abdominal intenso. La tomografía computarizada abdominal demostró la presencia de un aneurisma de la aorta infrarrenal, con un proceso inflamatorio que involucra y lo adhiere a la tercera porción del duodeno. El paciente solicitó el alta voluntaria ante la propuesta quirúrgica. Cinco días después reingresó en el Servicio de Terapia Intensiva por presentar hemorragia digestiva alta manifestada por hematemesis y melena de 48 horas de evolución, cuadro de shock hipovolémico, tensión arterial media (TAM) $30 \mathrm{mmHg}$, frecuencia cardíaca (FC): $140 \mathrm{lpm}$ y hematocrito del 18\%. Una vez compensado el trastorno hemodinámico, se realizó fibroendoscopia digestiva alta, constatándose sangrado activo en cara posterior de la tercera porción duodenal. Se decidió entonces la intervención quirúrgica por resangrado masivo, con el hallazgo intraoperatorio de aneurisma de aorta abdominal infrarrenal sacular adherido a la cara posterior de la tercera porción duodenal con comunicación a la luz de dicho órgano por un orificio de unos $4 \mathrm{~cm}$ de diámetro. Se realizó un by-pass aorto-bifemoral. Debido al grave compromiso hemodinámico presentó paro cardiorrespiratorio en quirófano, falleciendo pocas horas después en el Servicio de Terapia Intensiva.

\section{Caso clínico 2}

Presentamos el caso de un paciente de 49 años de edad, de sexo masculino; 4 meses antes del ingreso en nuestro hospital se le practicó cirugía vascular con reemplazo aorto-biilíaco por aneurisma aórtico abdominal infrarrenal.

Una semana previa al ingreso en el Servicio de Terapia Intensiva presentó episodios de proctorragia cuyos volúmenes fueron en aumento. Tras descompensación hemodinámica secundaria se indicó su ingreso en el Servicio de Terapia Intensiva. Refería además la presencia de dolor cólico periumbilical e hipogástrico de gran intensidad, hematocrito de $13 \%$ y sangrado incoercible, por lo que se indicó cirugía abdominal de urgencia ante la sospecha de una FAE, ya que la ecografía abdominal practicada horas antes informó imagen lineal superior a la cicatriz umbilical compatible con prótesis vascular, con zona hipoecogénica de $87 \times 67 \times 71 \mathrm{~mm}$ rodeándola. Se realizó laparotomía exploradora, constatándose hematoma retroperitoneal circundante a la prótesis previa y FAE con yeyuno a $60 \mathrm{~cm}$ del asa fija y colon sigmoideo. Presentó paro cardiorrespiratorio y óbito en la sala de operaciones.

\section{Caso clínico 3}

Se trata de un paciente de 51 años de edad, con diagnóstico de hipertensión arterial de más de 10 años de evolución y antecedente de cirugía de disección de aorta tipo B de la clasificación de Stanford, con colocación de prótesis de teflón, cuatro años antes de la consulta.

Una semana previa a su ingreso a Terapia Intensiva presentó dos episodios de melena y lipotimia. Ingresó con hematemesis abundante y melena y shock hipovolémico, siendo estabilizado con fluidos. Un nuevo episodio de hematemesis masiva lo lleva al paro cardiorrespiratorio y tras una adecuada resucitación se decidió la intervención quirúrgica, con el hallazgo quirúrgico de FAE al nivel de la tercera porción duodenal, y un orificio de $2 \times 1,5 \mathrm{~cm}$ de diámetro. Se realizó cierre simple, gastroyeyuno anastomosis y anastomosis coledocoduodenal. Falleció en el postoperatorio inmediato.

\section{DISCUSIÓN}

La descripción original de las FAE primarias fue realizada en 1817 y desde entonces se han comunicado alrededor de 230 casos $^{1}$. Los estudios de autopsias han mostrado que alrededor del 3,5\% de los episodios de hemorragias gastrointestinales altas masivas son debido a FAE primarias ${ }^{2}$. En otras series, en cambio, se dan valores de $0,04 \%$ a $0,07 \% \%^{3,12}$. La clásica tríada clínica de presentación de la FAE primaria, con dolor abdominal e irradiación lumbar, asociado a masa pulsátil abdominal y hemorragia intestinal más o menos grave que cesa espontáneamente, es poco frecuente. El tiempo que transcurre entre el primer sangrado y el evento catastrófico excede una semana en más del $40 \%$ de los casos ${ }^{7}$.

En una revisión de 118 pacientes el dolor abdominal estuvo presente en un $32 \%$, una masa abdominal pulsátil en menos del $25 \%$ y el sangrado intestinal como síntoma inicial en un 64\% de los casos ${ }^{8}$.

Con el paso de los años, y asociado al comienzo de las cirugías vasculares, se observó un aumento de la incidencia de las FAE secundarias. En el 90\% de los casos estas FAE son secundarias a comunicación entre el intestino y una prótesis vascular, complicación que se manifiesta en 0,6\%-2,3\% de los pacientes con injerto aórtico, pudiendo presentarse a los pocos meses de su implantación y hasta 15 años más tarde $^{3}$. Generalmente las FAE secundarias se caracterizan por sangrado intermitente de varios meses de evolución, que no siempre es masivo, manifestándose por melena, proctorragia o enterorragia, como ocurrió en el segundo y tercer caso presentados. Dado que se trata de una entidad que conlleva una elevada mortalidad, se requiere una alta sospecha clínica y una conducta quirúrgica urgente para mejorar el pronóstico de esta infrecuente patología?.

Tanto en las FAE primarias como en las secundarias la localización del trayecto fistuloso puede establecerse con cualquier porción del tubo digestivo, siendo más frecuente en el duodeno en alrededor del $80 \%$ de los casos (el 60\% incluyen a la tercera porción, del $5 \%$ al $8 \%$ a la cuarta, el $3 \%$ a la segunda y el $1 \%$ a la primera). La localización menos común es la colónica, como la hallada en el segundo caso que presentamos ${ }^{4}$. El yeyuno y el íleon están comprometidos en cerca del $4 \%$ de los $\operatorname{casos}^{5}$. 
La arterosclerosis es la causa patogénica presente en alrededor de dos tercios de los casos de las FAE primarias $^{2,3}$; otras causas incluyen adenocarcinoma pancreático, litiasis vesicular, diverticulitis, apendicitis, cuerpos extraños, aortitis sifilítica, tuberculosis, salmonelosis, infecciones bacterianas mixtas y fúngicas, aneurisma aórtico postraumático, tratamiento radiante, etc. En aquellos pacientes con aneurisma aórtico ateroesclerótico, sin implante protésico y sin infección demostrable, la constante presión/pulsación de la pared aneurismática presumiblemente erosionaría la pared intestinal provocando la comunicación entre dichos órganos ${ }^{6}$.

La fisiopatología de las FAE secundarias es controvertida. Se postula que serían multifactoriales con intervención de infección del material protésico, estrés mecánico de la prótesis y erosiones de la pared intestinal.

Según Giménez Bascuñana et al en el momento de la hemorragia muchos injertos están infectados, y los hemocultivos son positivos hasta en un $85 \%$ para gérmenes intestinales ${ }^{3}$. Estos datos comenzaron a observarse como un problema emergente a partir de la descripción inicial de Chalmers et al en 1993.

La fibroendoscopia digestiva alta es el método de estudio de primera elección, aunque tenga una especificidad del $25 \%$ al $50 \%$. La dificultad de encontrar el sitio exacto de sangrado por vía endoscópica se debe: a) al gran volumen de sangre volcado dentro del tracto intestinal superior; b) el endoscopio no puede alcanzar zonas más distales, ya que la mayoría de las FAE comprometen la tercera porción del duodeno como resultado de la proximidad anatómica con la arteria aorta, y c) el hallazgo de otras lesiones potencialmente sangrantes.

La introducción de los modernos enteroscopios ha permitido el examen directo de áreas cada vez más extensas de la luz intestinal. La laparotomía exploradora con enteroscopia intraoperatoria empezó a utilizarse como método para el diagnóstico de sangrado intestinal oculto hace ya algunos años, y constituye el método de elección en pacientes con sangrado intestinal grave e inestabilidad hemodinámica con sospecha de FAE, en los que una rápida actuación es esencial ${ }^{10,11}$. La angiografía convencional es de utilidad limitada, especialmente si el sangrado es lento. Otros estudios como ecografía, estudios radioisotópicos, tomografía computarizada, resonancia magnética, etc., tienen una apreciable utilidad cuando el sangrado no es masivo.
Estos pacientes presentan alta morbimortalidad perioperatoria. En una reciente revisión de FAE primarias se concluye que alrededor de dos tercios de los pacientes murieron durante la cirugía o dentro de los 30 días del postoperatorio ${ }^{9}$. No hemos hallado datos publicados sobre mortalidad en las FAE secundarias. Esta desfavorable evolución se ha evidenciado en los pacientes de nuestra serie.

\section{Declaración de conflicto de intereses}

Los autores han declarado no tener ningún conflicto de intereses

\section{BIBLIOGRAFÍA}

1. Farber A, Grigoryants V, Palac DM, Chapman T, Cronenwett JL, Powell RJ. Primary aortoduodenal fistula in a patient with a history of intravesical therapy for bladder cancer with bacillus Calmette-Guérin: review of primary aortoduodenal fistula without aortic aneurysm. J Vasc Surg. 2001;33:868-73.

2. Brock RC. Aortic homografting: a report of six successful cases. Guys Hosp Rep. 1953;1:204-28.

3. Giménez Bascuñana A, Nieto Olivares A, Acosta Ortega J, Piñero Madrona A, MonzonesTorres M, Bermejo López. Fístula aortoentérica. Una causa inusual de hemorragia digestiva. Gastroenterol Hepatol. 1995;18:22-8.

4. Champion MC, Sullivan SN, Coles JC. Aortoenteric fistula. Incidence, presentation, recognition and management. Ann Surg. 1982;195:314-7.

5. Sevastos N, Rafailidis P, Kolokotronis K, Papadimitriou K, Papatheodoridis GV. Primary aortojejunal fistula due to foreign body: a rare cause of gastrointestinal bleeding. Eur J Gastroenterol Hepatol. 2002;14:797-800.

6. O'Mara CS, Williams MG, Emst CB. Secondary aortoenteric fistula. A 20 year experience. Am J Surg. 1981;142:203-9.

7. Mollerup CL, Strand L. Gastrointestinal haemorrhage due to fistula from aortic aneurysm. Acta Chir Scand. 1987;153:6312

8. Sweeney MS, Gadacz TR. Primary aortoduodenal fistula: manifestation, diagnosis, and treatment. Surgery. 1984; 96:492-7.

9. Finch L, Heathcock RB, Quigley T, Jiranek G, Robinson D. Emergent Treatment of a Primary Aortoenteric Fistula with Nbutyl 2-cyanoacrylate and Endovascular Stent. J Vasc Interv Radiol. 2002;13:841-3.

10. Lau WY. Intraoperative enteroscopy: indications and limitations. Gastrointest Endosc. 1990;36:268-71.

11. Ress AM, Benacci JC, Sarr MG. Efficacy of intraoperative enteroscopy in diagnosis and prevention of recurrent, occult gastrointestinal bleeding. Am J Surg. 1992;163:94-8.

12. Voorhoeve R, Moll FL, de Letter JA, Bast TJ, Wester JP, Slee PH. Primary aortoenteric fistula: report of eight new cases and review of the literature. Ann Vasc Surg. 1996;10:40-7. 